\title{
Discovery of potential anti-infectives against Staphylococcus aureus using a Caenorhabditis elegans infection model
}

\author{
Cin Kong ${ }^{1}$, Wageeh A Yehye ${ }^{2}$, Noorsaadah Abd Rahman ${ }^{3}$, Man-Wah Tan ${ }^{4,5}$ and Sheila Nathan ${ }^{\text {1* }}$
}

\begin{abstract}
Background: The limited antibiotic options for effective control of methicillin-resistant Staphylococcus aureus infections has led to calls for new therapeutic approaches to combat this human pathogen. An alternative approach to control MRSA is through the use of anti-infective agents that selectively disrupt virulence-mediated pathways without affecting microbial cell viability or by modulating the host natural immune defenses to combat the pathogen.

Methods: We established a C. elegans - S. aureus liquid-based assay to screen for potential anti-infectives against S. aureus. The assay was utilized to screen 37 natural extracts and 29 synthetic compounds for the ability to extend the lifespan of infected nematodes. Disc diffusion and MIC microdilution tests were used to evaluate the anti-microbial properties of these natural extracts and synthetic compounds whilst in vivo bacterial CFU within the C. elegans gut were also enumerated.

Results: We screened a total of 37 natural extracts and 29 synthetic compounds for anti-infective properties. The screen successfully revealed 14 natural extracts from six plants (Nypa fruticans, Swietenia macrophylla, Curcuma longa, Eurycoma longifolia, Orthosiphon stamineus and Silybum eburneum) and one marine sample (Faunus ater) that improved the survival of S. aureus-infected worms by at least 2.8-fold as well as 14 synthetic compounds that prolonged the survival of $S$. aureus-infected nematodes by 4 -fold or greater. An anti-microbial screen of all positive hits demonstrated that $8 / 28$ hits had no effect on $S$. aureus growth. Of these 8 candidates, 5 of them also protected the worms from MRSA infection. We also noted that worms exposed to $N$. fruticans root and $O$. stamineus leaf extracts showed reduced intestinal colonization by live $S$. aureus. This suggests that these extracts could possibly activate host immunity to eliminate the bacteria or interfere with factor/s that prevents pathogen accumulation.
\end{abstract}

Conclusion: We have successfully demonstrated the utility of this liquid-based screen to identify anti-infective substances that prolong S. aureus-infected host survival without affecting bacterial cell viability.

Keywords: S. aureus, C. elegans, Anti-infectives

\section{Background}

Staphylococcus aureus is capable of causing a variety of human diseases in both hospital and community settings. Diseases caused by $S$. aureus range from superficial skin infections to life threatening systemic bacteremia [1]. The effectiveness of current antibiotic treatments that interfere with $S$. aureus growth and viability is limited by the development of drug resistance. The rapid emergence of

\footnotetext{
* Correspondence: sheila@ukm.my

'School of Biosciences and Biotechnology, Faculty of Science and Technology, Universiti Kebangsaan Malaysia, 43600 UKM Bangi, Selangor, Malaysia

Full list of author information is available at the end of the article
}

antibiotic resistant strains [2,3] highlights the urgent need for newer and safer strategies to combat infection. An alternative approach to overcome the pitfalls of drug resistance is to develop anti-infective agents that selectively disrupt virulence-mediated pathways without affecting microbial cell viability [4-6] or by modulating the host natural immune defenses to combat the pathogen [7].

A number of different strategies have been adopted to identify new anti-infectives and these include using wholeanimal infection models such as Caenorhabditis elegans [8-12]. Whilst traditional in vitro and whole cell drug screens are acknowledged as the established paradigm for

\section{Ciomed Central}


identifying antimicrobial molecules, the whole animal approach allows for early and direct assessment of in vivo drug efficacy, thus, eliminating compounds that are toxic to the host or with poor pharmacokinetic properties [13]. As current understanding of host-pathogen interactions and bacterial pathogenesis continues to increase $[14,15]$, the C. elegans model presents an advantage in being able to simultaneously identify compounds that target bacterial virulence as well as host defense.

In a live animal model with a recognized host-pathogen interaction, potential hits that selectively disrupt the virulence pathways utilized by pathogens to establish infections can be identified $[13,16]$. Furthermore, several conserved innate immune signaling pathways have been revealed from studies of host-pathogen interactions using C. elegans [17]. Conceptually, anti-infectives that target nonessential genes are likely to impose a lower level of selective pressure and probability of resistance development [18] whilst ensuring the preservation of host endogenous microflora.

Infection of $C$. elegans by $S$. aureus is a robust platform to elucidate the mechanisms of host-pathogen interaction [19-22]. In this study, our aim was to extend these studies to the discovery of novel anti-infectives. To achieve this objective, we chose to establish a $C$. elegans - S. aureus liquidbased screen to identify anti-infectives that extend the lifespan of S. aureus - infected nematodes from a collection of locally acquired natural products and synthetic compounds.

\section{Methods}

\section{Bacterial and nematode strains}

The methicillin-susceptible $S$. aureus strain NCTC83254 [19] and methicillin-resistant $S$. aureus (MRSA) strain ATCC33591 were grown with aeration in Trypticase Soy (TS) media (Oxoid/Pronadisa) at $37^{\circ} \mathrm{C}$ while Escherichia coli strain OP50 was grown in Luria Bertani (LB) broth supplemented with streptomycin $(100 \mu \mathrm{g} / \mathrm{mL})$. The wild type C. elegans Bristol N2 hermaphrodite strain was propagated on nematode growth medium (NGM) and fed on the standard laboratory food source, E. coli OP50. The animals were age-synchronized by bleaching with alkaline hypochlorite and sodium hydroxide to release embryos. Embryos were placed on plates containing concentrated E. coli $\mathrm{DH} 5 \alpha$ at $25^{\circ} \mathrm{C}$ until the worms reached the young adult stage. To eliminate the confounding effects of reproduction on the scoring of surviving worms, wild type C. elegans were made sterile by RNAi knockdown of the pos-1 gene which resulted in worms laying unhatched eggs [23]. The pos-1 RNAi treated worms were grown for $\sim 45 \mathrm{hrs}$ at $25^{\circ} \mathrm{C}$ until they reached young adult stage and were ready to be used in the screen.

\section{Preparation of extracts and compounds}

A total of 18 plant species and one marine sample were the source of the natural extracts. Extracts UE-01-1 to
UE-08 were extracted according to the previously described method [24]. Briefly, the plant materials and animal sample were collected from several locations in Malaysia. The samples were authenticated, deposited and catalogued with specimen voucher numbers (Additional file 1). All samples were then air-dried, powdered and extracted with polar and non-polar solvents. Solvents were then removed by evaporation using a rotary evaporator. Extracts UE-09 to UE-20 (except UE-15) were prepared by Herbal Science Pte. Ltd., a local pharmaceutical company. In brief, the raw materials were collected and quality assessed through a series of stringent microbial tests as well as heavy metals test using an Atomic Absorption Spectrophotometer (AAS). They were then dried, comminuted or ground into fine powder before water-based extraction was performed. The microbial and trace metal element tests were repeated again on the yield obtained. The finished products were examined for the presence of certain functional groups/molecules using Fourier transform infrared spectroscopy (FTIR) and High-performance liquid chromatography (HPLC). UE-15 was purchased from Merck, Germany.

Four different series of butylated hydroxytoluene (BHT) derivatives (Figure 1) were designed and synthesized by the Chemistry Department, University of Malaya, Malaysia. Thiosemicarbazides were synthesized according to the method of Yehye et al. [25,26]. 1,3,4thiadiazoles and 1,2,4-triazole bearing the free radical scavenger BHT were synthesized using acid-(base-) catalyzed intramolecular dehydrative cyclization reaction of the 1-acylthiosemicarbazides whilst hydrazones were synthesized from the reaction between free amino groups and properative aldehydes (Yehye et al., in preparation). All extracts and compounds were dissolved in dimethyl sulfoxide (DMSO), filtered through a $0.2 \mu \mathrm{m}$ membrane filter and stored at $-20^{\circ} \mathrm{C}$. Detailed information on all natural

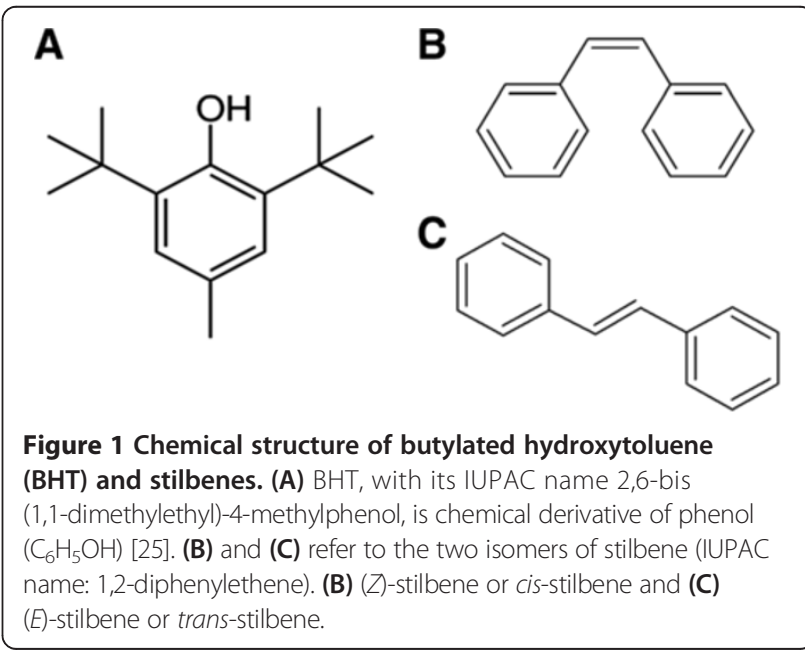


extracts and synthetic compounds used in this study is provided in Additional file 2.

\section{C. elegans survival assay}

For the agar-based assay, $10 \mu \mathrm{L}$ of a $S$. aureus overnight culture in TS broth was spread on $3.5 \mathrm{~cm}$ TS agar plates and incubated at $37^{\circ} \mathrm{C}$ for $24 \mathrm{hrs}$. The plates were then allowed to equilibrate to room temperature for at least 1 hour before use. A total of 40 young adult worms were transferred to the agar plate and incubated at $25^{\circ} \mathrm{C}$. Worm mortality was monitored at 24-hour intervals. E. coli OP50 on NGM was used as the negative control. Three independent experiments were performed.

For the liquid-based assay, a 24-well plate was filled with S. aureus/MRSA in a liquid medium (80\% M9 buffer, $20 \%$ S. aureus overnight culture and $10 \mu \mathrm{g} / \mathrm{mL}$ cholesterol) and 20 synchronized young adult nematodes were transferred into each well. Wells containing C. elegans, E. coli OP50 and M9 buffer served as the control. The total number of worms for each treatment was 120 in six wells representing six technical replicates. The plate was incubated at $25^{\circ} \mathrm{C}$ and survival was monitored every $24 \mathrm{hrs}$ following exposure to the pathogen.

\section{Anti-infective screening}

Screening was performed in liquid medium in a 24 -well plate. The liquid screening medium consisted of worm M9 buffer and overnight culture of $S$. aureus/MRSA in TS broth with $10 \mu \mathrm{g} / \mathrm{mL}$ of cholesterol at a ratio of $4: 1$ $(\mathrm{v} / \mathrm{v})$. The medium was supplemented with either natural extracts or synthetic compounds to a final concentration of $200 \mu \mathrm{g} / \mathrm{mL}$ (natural extracts) and $200 \mu \mathrm{M}$ (synthetic compounds). All components of the assay were mixed evenly and $500 \mu \mathrm{L}$ of medium was transferred into each well in a 24-well plate. In control wells, the extract or synthetic compound was replaced with $1 \%$ DMSO or $S$. aureus was replaced by E. coli OP50 as the nematode food source. Each extract or compound was tested in triplicate wells. Approximately 10-15 age-synchronized pos-1 treated young adult stage N2 nematodes were transferred into each well and the plate was incubated at $25^{\circ} \mathrm{C}$. Worms treated with pos- 1 RNAi produced unhatched eggs thereby eliminating potential complications during the scoring of worms. Worm survival was scored manually every 24 hrs for 5 days. In addition, turbidity of the extract/compound containing media was also observed and recorded. An extract or compound was considered a positive hit if $>50 \%$ of the infected worms survived compared to $\leq 20 \%$ survival in the untreated control worms in least two replicates. All positive hits from the primary screen were subjected to a secondary screen for confirmation. A summary of the one-step anti-infective liquid screen approach used in this study is presented in Additional file 3 and is compared to the previously reported two-step agar-liquid protocol used by Moy et al. [8] for screening of antimicrobials against Enterococcus faecalis and high throughput liquid-based chemical screen to screen for molecules that attenuate Pseudomonas aeruginosa virulence and rescue C. elegans from infection [11].

\section{Determination of antimicrobial property by disc diffusion assay}

The disc diffusion method [27] was used to determine if the extracts and compounds also promoted antimicrobial features. Sterile filter paper discs (Whatman No. 1, $6 \mathrm{~mm}$ ) were impregnated with each of the extracts $(20 \mathrm{mg} / \mathrm{mL})$ or compounds $(20 \mathrm{mM})$ and left to dry under sterile conditions overnight. S. aureus was spread evenly onto the surface of TS agar plates using glass beads before the discs were positioned on the inoculated agar surface. The bacterial inoculum size was standardized at $10^{6} \mathrm{cfu} / \mathrm{mL}$ by adjusting the optical density of the bacterial suspension. DMSO served as the negative control with gentamicin $(200 \mu \mathrm{g} / \mathrm{mL})$ as the positive control. All plates were incubated for $18 \mathrm{hrs}$ at $37^{\circ} \mathrm{C}$. Antibacterial activity was confirmed by the presence of a clear zone of inhibition around the disc.

\section{Determination of MIC and MBC}

The bacteriostatic and bactericidal effects of all positive extracts and compounds were evaluated using the broth microdilution minimum inhibitory concentration (MIC) assay [28]. A range of different concentrations of extract/ compound was prepared in a 48-well plate, followed by inoculation with $10^{6} \mathrm{cfu} / \mathrm{mL}$ S. aureus. The plate was then incubated at $37^{\circ} \mathrm{C}$ for $18 \mathrm{hrs}$. Gentamicin, the standard antibiotic for $S$. aureus infections, was used as a control. All extracts/compounds were assayed in triplicate. The MIC endpoint recorded was the lowest concentration of the extract/compound used in wells that showed no turbidity after $18 \mathrm{hrs}$ incubation, indicating an absence of bacterial growth. The presence or absence of turbidity was confirmed by absorption readings at $600 \mathrm{~nm}$. The minimum bactericidal concentration $(\mathrm{MBC})$ was determined by spreading the culture from the respective wells on sterile agar plates. The MBC value was the lowest concentration of extract/compound used where no apparent growth of bacteria was observed on the agar plate.

\section{Enumeration of bacterial colony forming units (CFU) within the $C$. elegans gut}

After 24 hours exposure to $S$. aureus in liquid medium in the presence and absence of extracts/compounds, 10-12 live worms were randomly picked and briefly anesthetized in $25 \mathrm{mM}$ Levamisole. The worms were washed at least twice in $200 \mu \mathrm{L}$ antibiotic cocktail comprising $25 \mathrm{mM}$ Lev and gentamicin $10 \mu \mathrm{g} / \mathrm{mL}$ followed by incubation for 
45 min to 1 hour to completely kill bacterial cells associated with the worm cuticle. Then, the worms were washed three times with $200 \mu \mathrm{L}$ of $25 \mathrm{mM}$ Lev to eliminate the killed bacteria and residual antibiotic. The number of worms were recorded followed by mechanical disruption in $50 \mu \mathrm{L}$ of $1 \%$ Triton X (Sigma-Aldrich, USA; X100) using a motorized pestle. Serial dilutions were performed on the worm lysates and $10 \mu \mathrm{L}$ of each dilution was spotted on TS agar supplemented with $5 \mu \mathrm{g} / \mathrm{mL}$ nalidixic acid. Colonies were counted after incubating the plates at $37^{\circ} \mathrm{C}$ overnight. Bacterial CFU per worm was calculated using the formula reported by Ooi et al. [29].

\section{Statistical analysis}

All data are presented as mean \pm standard deviation (SD) of at least two independent experiments. All screening assays, antibacterial tests and in vivo CFU assay were replicated in a comparable manner. Data from the killing assays were analyzed with StatView ${ }^{\bullet}$ 5.0.1 (SAS Institute, Inc) and plotted using the Kaplan-Meier Cumulative Survival Plot for Time (nonparametric survival analysis). The pair wise comparison was analyzed using the Log-rank (Mantel-Cox) significance test.

\section{Results}

\section{Liquid-based screen was able to detect more hits}

Most studies on C. elegans infections by $S$. aureus are routinely performed on agar plates. In this study, we observed significant reduction in the lifespan of $C$. elegans infected by $S$. aureus in both the agar-based and liquidbased assays (Figure 2A and B). Specifically, the mean time to death $\left(\mathrm{TD}_{\text {mean }}\right)$ for $S$. aureus-infected animals assayed on agar was $73 \pm 1.5$ hrs which was consistent with previous studies [20]. Similarly, in the liquid-based assay, worms in M9 buffer and $S$. aureus culture (4:1, v/v) had a $\mathrm{TD}_{\text {mean }}$ of $72 \pm 5$ hrs. $S$. aureus required 4 to 5 days for complete killing of $C$. elegans. In liquid medium, the worms infected by $S$. aureus started to die after 24 hrs exposure to the pathogen. Mobility, pharyngeal pumping and foraging rates of worms exposed to pathogens progressively decreased with time until all worms became immobile and died. The results confirmed that in liquid medium, $S$. aureus is able to kill the nematodes as efficiently as the standard agar-based assay system.

To establish the utility of the liquid assay for antiinfective screening, we screened a subset of 18 natural extracts using both liquid- and agar-based assays. In the agar-based assay, extracts were added into the TS agar whilst the prepared agar was in a molten state. Nematode survival on extract-added plates was compared to the control (agar without extract). Only 1/18 demonstrated potential anti-infective properties when compared to controls in the absence of extract on solid agar (Figure 3). By contrast, when the assay was performed in the liquid-based assay, we were able to detect $7 / 18$ potential hits that reproducibly extended the lifespan of $S$. aureus-infected worms in at least two independent experiments (Figure 3). These results indicated that screening using a liquid-based assay was able to detect more hits than the conventional agarbased assay. Thus, we carried out the anti-infective screen on the rest of the extracts and compounds using the liquid-based screen.

Direct comparison of the hits identified from both the liquid and agar based screens (Figure 3) confirmed that only UE-08 promoted the survival of infected worms in both agar-based and liquid based screens. UE-01-1, UE-01-2, UE-01-4, UE-01-6, UE-01-7 and UE-03-5 were able to improve the lifespan of infected worms in liquid medium but not in agar and this could be due to thermal degradation of certain active molecules (saponins, flavonoids) during
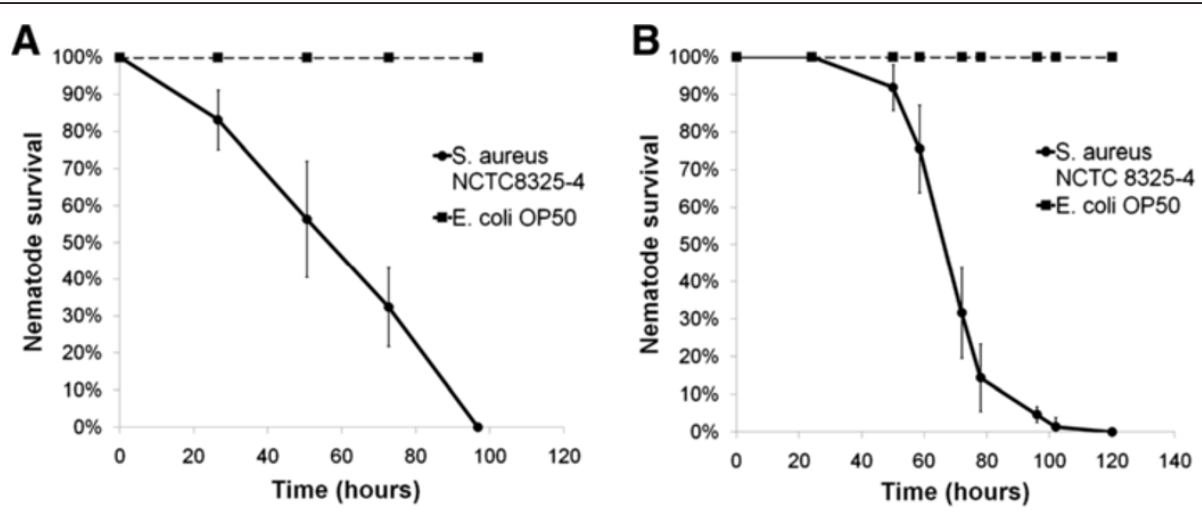

Figure 2 S. aureus kills C. elegans in both liquid and agar medium. Representative survival curves of C. elegans following infection by S. aureus strain NCTC8325-4 in (A) liquid medium consisting of 80\% M9 buffer, 20\% S. aureus overnight culture and cholesterol and (B) TS agar medium. Graph (A) shows the mean \pm SD of six replicates (20 nematodes/replicate) and graph (B) presents the mean \pm SD of three replicates (40 nematodes/replicate) from a representative of three independent assays. 


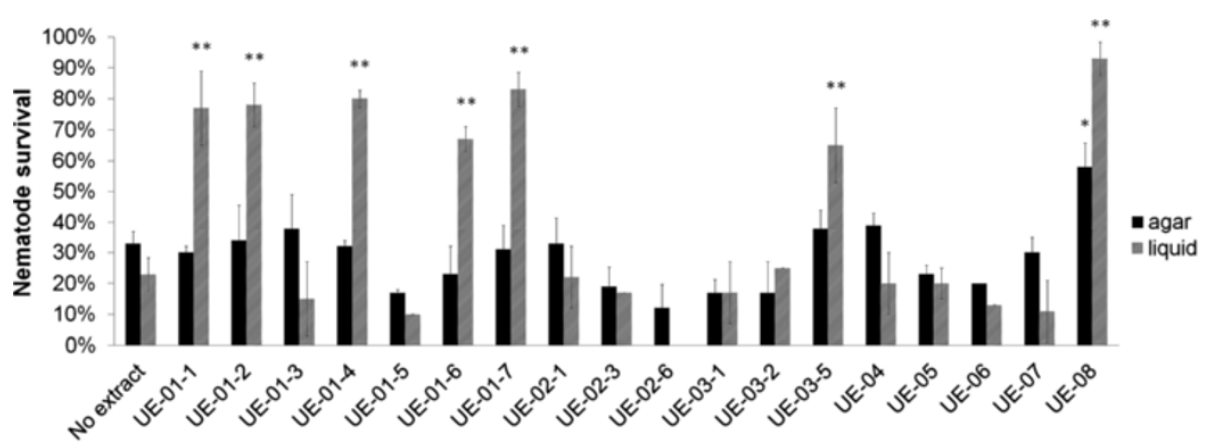

Figure 3 Liquid-based assay identified more hits than the agar-based assay. Comparison of hits from the preliminary screen of 18 natural extracts on both agar-based and liquid-based assays. All natural extracts were tested at same concentration $(200 \mu \mathrm{g} / \mathrm{mL})$ in both agar and liquid assays. The graph represents the mean percentage of survival \pm SD at 72 hours post-infection of three replicates from a representative of two individual experiments. The survival of extract-treated worms is compared to the control (no extract). (*) indicates positive in agar-based screens while $\left(^{* *}\right)$ indicates positive in liquid-based screens.

the media preparation. Further comparisons between the solid agar and liquid-based screens are elaborated in Table 1.

\section{Selected extracts and compounds enhanced the survival of S. aureus-infected nematodes}

We performed a small scale screen in 24-well plates using the S. aureus - C. elegans liquid infection assay to identify more potential anti-infectives that improved the survival of infected worms. Screening over 37 natural plant and marine invertebrate extracts and 29 synthetic compounds revealed a total of 14 natural extracts and 14 synthetic compounds that conferred increased survival of S. aureus-infected nematodes (Figure 4). The criteria adopted to classify an extract or compound as a positive hit was the survival of $>50 \%$ of the infected worms compared to $\leq 20 \%$ survival in untreated wells over at least two replicates. All positive hits contributed to more than $60 \%$ C. elegans survival following infection and treatment for 72 hrs compared to about $20 \%$ survival in untreated controls (Table 2). All positive natural extracts increased nematode survival by 2.8 -fold or more while all positive synthetic compounds exhibited a more significant increase in survival of at least 4-fold. The worms fed on E. coli OP50 remained alive throughout the assay. Detailed information on all positive hits is shown in Table 2.

The striking differences in the appearance of live worms and worms that succumbed to infection facilitated the screening process. The infected worms were generally smaller in size as compared to healthy worms fed on E. coli OP50. Dead worms did not move or exhibit muscle tone and did not respond to external stimuli. They appeared as straight rigid rods in the medium with no observable pharyngeal pumping (Figure 5A). In contrast, worms exposed to E. coli OP50 maintained a sinusoidal 'S' shape and moved actively. These worms grew in size and developed into gravid adults which produced eggs (Figure 5B). With the addition of extract/ compound that exhibited potential anti-infective properties, the worms were rescued from the $S$. aureus infection as demonstrated by their ability to overcome the debilitating effects of the infection. As indicated in Figure $5 \mathrm{C}$, the surviving infected nematodes appear to be smaller in size, similar to that of the untreated infected worms. Nevertheless, the live worms were able to maintain the sinusoidal ' $\mathrm{S}$ ' shape posture and respond to external stimuli when touched. In addition, the worms

Table 1 Comparison between agar-based versus liquid-based screens

\begin{tabular}{lll}
\hline Criteria & Agar-based & Liquid-based \\
\hline Positive hits identified & 1 out of 18 natural extracts screened & 7 out of 18 natural extracts screened \\
Average time for media preparation & $2-3$ days & $<30$ minutes \\
Possibility of compound degradation & $\begin{array}{l}\text { Addition of compound into the molten agar at high } \\
\text { temperature may cause compound degradation }\end{array}$ & $\begin{array}{l}\text { Does not involve high temperature. Thus, } \\
\text { degradation of compound is not possible }\end{array}$ \\
Exposure to compound & $\begin{array}{l}\text { Compound is restricted to the agar media, resulting } \\
\text { in limited worm exposure to the compound }\end{array}$ & $\begin{array}{l}\text { Worms are bathed in a homogenous } \\
\text { solution of compound }\end{array}$ \\
Counting efficiency & $\begin{array}{l}\text { Time-consuming to locate the worms on a larger } \\
\text { surface area }\end{array}$ & $\begin{array}{l}\text { Smaller surface area and clear distinguishable } \\
\text { phenotype of the worms allows quick scoring }\end{array}$ \\
& Possible agar color changes complicate worm scoring & Colorless M9 buffer eases the scoring of worms
\end{tabular}




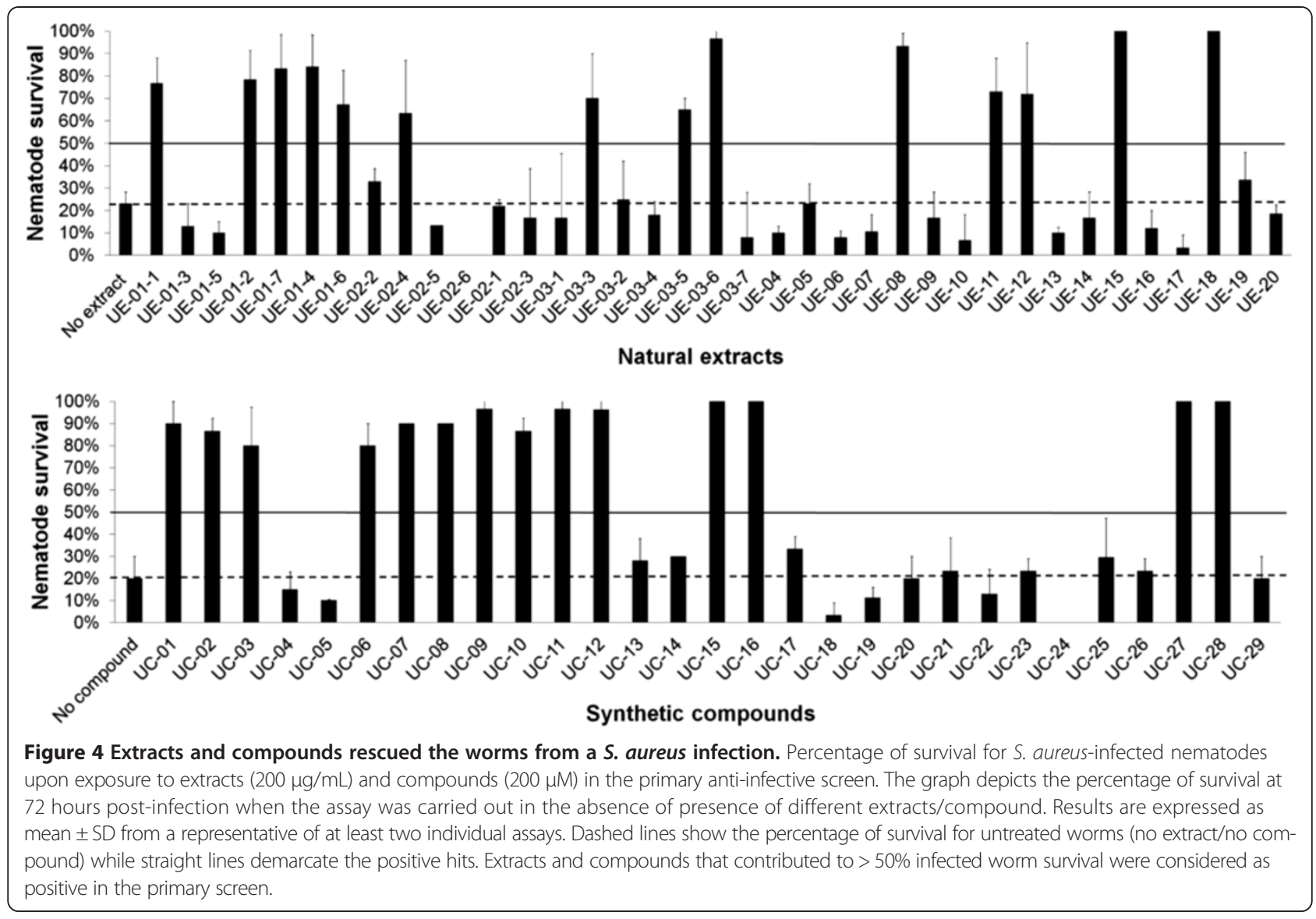

continued to move actively even after 72 hrs exposure to $S$. aureus. Although the worms were able to survive upon $S$. aureus infection in the presence of hit extracts/ compounds, most of the live worms appeared to experience delayed development as compared to healthy worms fed on E. coli OP50.

Among 18 plant species tested, extracts from six plants (N. fruticans, S. macrophylla, C. longa, E. longifolia, O. stamineus and S. eburneum) were able to extend the lifespan of $S$. aureus-infected animals. The marine sample (F. ater) also possessed potential anti-infective activity. N. fruticans, S. macrophylla, C. longa, E. longifolia and O. stamineus are commonly found in Malaysia and used widely by the local community as folk medicine. Hence, local traditional herbs are a promising source of largely unexplored classes of anti-infectives.

We tested seven samples from $N$. fruticans obtained from different parts of the plant through the use of different extraction solvents. Our screen identified 5/7 $N$. fruticans samples that increased the survival of infected nematodes by 2.9 to 3.7 fold. Different parts of the plant, for example, leaves, roots and husks, may contain different sets of bioactive compounds whilst the different extraction solvents used may also separate different bioactive constituents. Alternatively, the differences may be due to variations in the quantity of the individual active components in these tissues. Both $C$. longa and curcumin showed an equally strong effect by enhancing the survival of $S$. aureus-infected nematodes by at least 4-fold (Table 2). Curcumin is the main active ingredient in $C$. longa suggesting that curcumin might be the contributing bioactive component of C. longa. Methanol, butanol and ethyl acetate extracts of $S$. macrophylla were also able to prolong the lifespan of $S$. aureus-infected worms with the butanol extract showing the most enhanced effect, whereby worm survival increased by $4.2-$ fold. Of all the extracts tested, the $S$. macrophylla butanol extract, curcumin and S. eburneum seed extract demonstrated very promising anti-infective activity by promoting the survival of $S$. aureus-infected worms by more than 4-fold.

With the synthetic compounds, all 14 hits promoted the survival of infected worms by at least 4-fold with UC-15, UC-16, UC-27 and UC-28 demonstrating the most prominent effect, whereby worm survival increased by 5 -fold. Compounds UC-01, UC-02 and UC-03 are variations of thiosemicarbazide, UC-07, UC-08, UC-09, UC-10, UC-11 and UC-12 were synthesized based on of 1,2,4-triazole-5-thiones, UC-15 and UC-16 are derived from hydrazones, UC-27 and UC-28 are stilbene derivatives 
Table 2 Natural extracts and synthetic compounds that promote survival of S. aureus-infected nematodes

\begin{tabular}{|c|c|c|c|c|c|}
\hline No. & Extract/compound ID & Plant/animal species & Extraction solvent & Mean \% of survival & Fold change survival vs. untreated $^{a}$ \\
\hline 1 & UE-01-1 & Nypa fruticans husks & Methanol & 76.7 & 3.3 \\
\hline 2 & UE-01-2 & N. fruticans leaves & Hexane & 78.4 & 3.4 \\
\hline 3 & UE-01-4 & N. fruticans roots & Butanol & 84.2 & 3.7 \\
\hline 4 & UE-01-6 & N. fruticans roots & Chloroform & 67.3 & 2.9 \\
\hline 5 & UE-01-7 & N. fruticans leaves & Chloroform & 83.3 & 3.6 \\
\hline 6 & UE-02-4 & Faunus ater & Methanol & 63.3 & 2.8 \\
\hline 7 & UE-03-3 & Swietenia macrophylla seeds & Methanol & 70.0 & 3 \\
\hline 8 & UE-03-5 & S. macrophylla seeds & Ethyl acetate & 65.0 & 2.8 \\
\hline 9 & UE-03-6 & S. macrophylla seeds & Butanol & 96.7 & 4.2 \\
\hline 10 & UE-08 & Curcuma longa & Water & 93.3 & 4 \\
\hline 11 & UE-11 & Eurycoma longifolia roots & Water & 73.0 & 3.2 \\
\hline 12 & UE-12 & Orthosiphon stamineus leaves & Water & 71.8 & 3.1 \\
\hline 13 & UE-15 & Curcumin & - & 100.0 & 4.3 \\
\hline 14 & UE-18 & Silybum eburneum seeds & Water & 100.0 & 4.3 \\
\hline 15 & UC-01 & - & - & 90.0 & 4.5 \\
\hline 16 & UC-02 & - & - & 86.7 & 4.3 \\
\hline 17 & UC-03 & - & - & 80.0 & 4 \\
\hline 18 & UC-06 & - & - & 80.0 & 4 \\
\hline 19 & UC-07 & - & - & 90.0 & 4.5 \\
\hline 20 & UC-08 & - & - & 90.0 & 4.5 \\
\hline 21 & UC-09 & - & - & 96.7 & 4.8 \\
\hline 22 & UC-10 & - & - & 86.7 & 4.3 \\
\hline 23 & UC-11 & - & - & 96.7 & 4.8 \\
\hline 24 & UC-12 & - & - & 96.3 & 4.8 \\
\hline 25 & UC-15 & - & - & 100.0 & 5 \\
\hline 26 & UC-16 & - & - & 100.0 & 5 \\
\hline 27 & UC-27 & - & - & 100.0 & 5 \\
\hline 28 & UC-28 & - & - & 100.0 & 5 \\
\hline
\end{tabular}

${ }^{a}$ Note that $\sim 20 \%$ of the untreated worms survived 72 hours post-infection. The mean percentage of survival from one representative biological replicate out of three independent assays is presented.
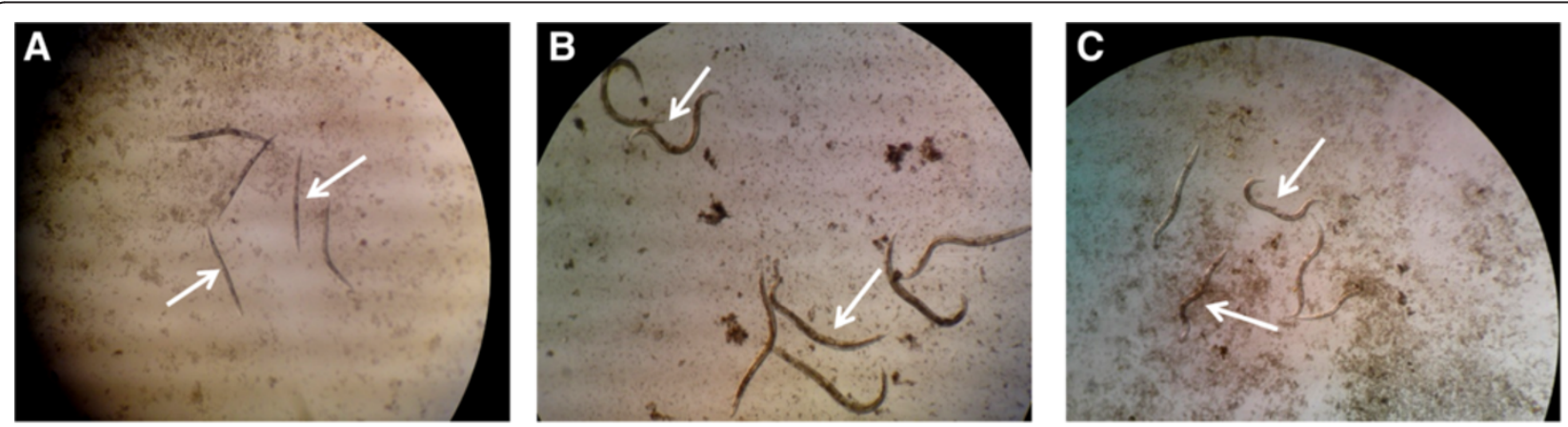

Figure 5 Distinct appearances of dead and alive C. elegans. Representative micrographs of C. elegans exposed either to S. aureus (A), E. coli OP50 (B) or S. aureus in the presence of extracts/compounds (C). The worms were examined under a stereomicroscope (Nikon SMZ645) at a magnification of $\times 40$. White arrows denote the worms. 
while UC-06 is a variant of 1,3,4-thiadiazole. With the exception of stilbene, all other compounds were fused to a BHT moiety. The structures of all 14 positive synthetic compounds are shown in Figure 6.

As illustrated in Figure 7, different extracts/compounds exhibited different degrees of protection in extending the lifespan of nematodes following S. aureus infection. During the screening process, we also took note of changes in media turbidity indicative of bacterial presence and growth. Upon addition of selected extracts and compounds, for example UE-08 and compound UC-15, into the medium, a significant clearing of the medium was noted.

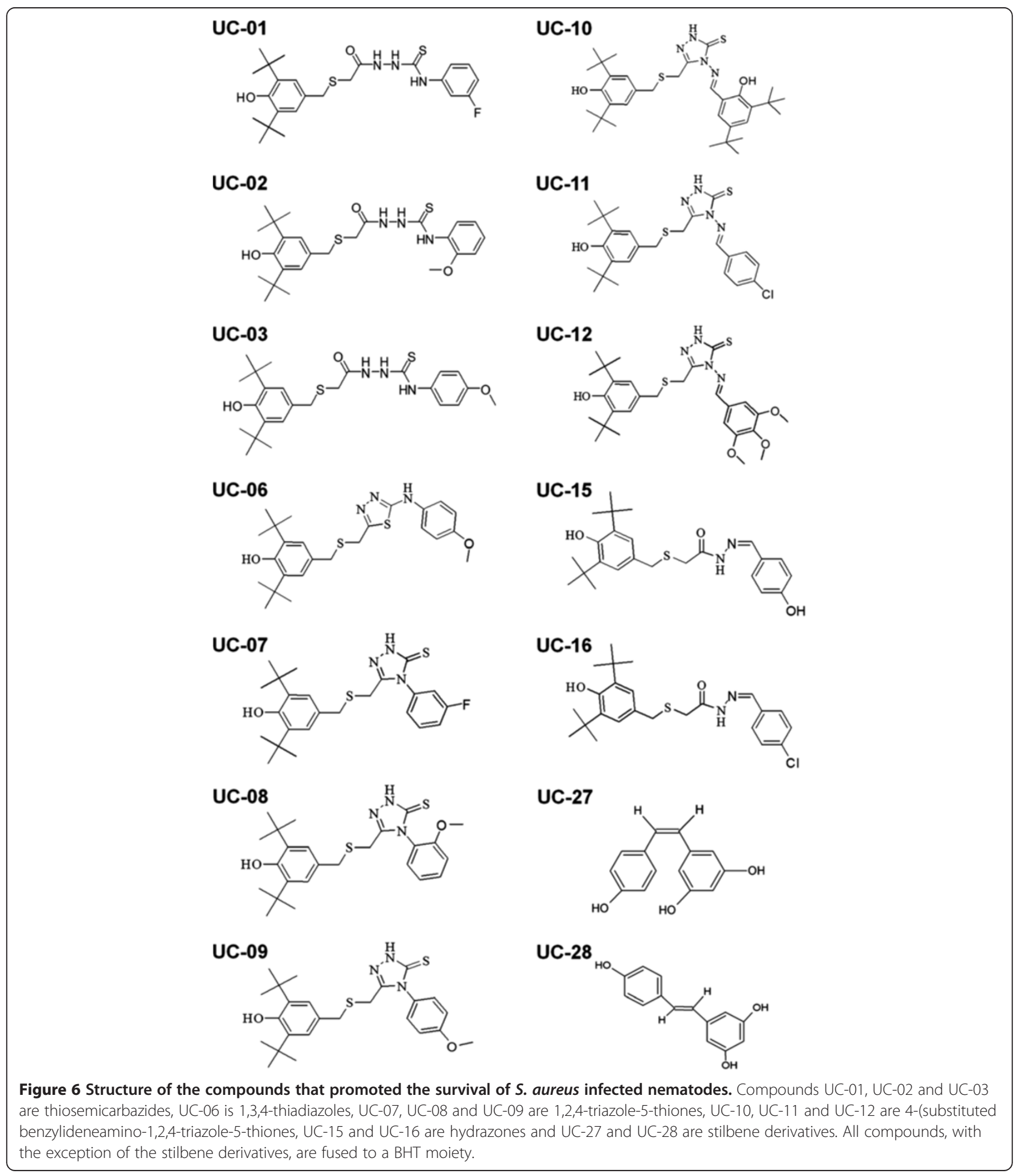



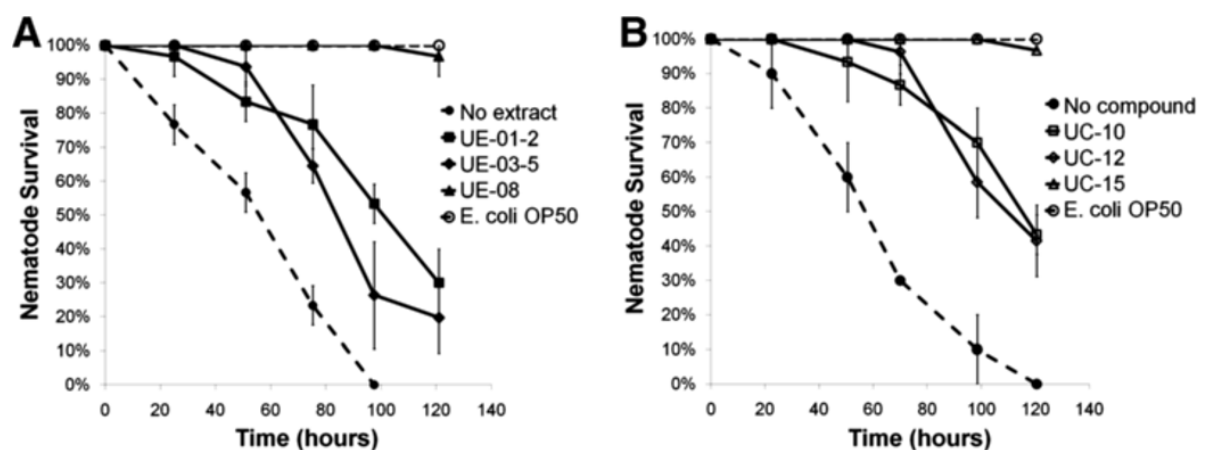

Figure 7 Survival of $C$. elegans is improved over time upon supplementation of extracts and compounds. (A and B) The survival curves of S. aureus-infected C. elegans upon exposure to selected hit extracts $(200 \mu \mathrm{g} / \mathrm{mL})$ and compounds $(200 \mu \mathrm{M})$. Enhanced survival of infected worms can be seen in the presence of extracts UE-01-2, UE-03-5, UE-08 (A) and compounds UC-10, UC-12 and UC-15 (B). Data at each time point are the average of three wells with each well containing approximately 10-15 animals per well. In a pair-wise comparison to no extract using log-rank tests, the difference between all of the treatments was significant $(p<0.0001)$.

When we correlated this observation to the MIC and MBC results of all positive hits as shown in Table 3, we noted that extract UE-08 and compound UC-15 possessed strong antibacterial activity against $S$. aureus. The survival curves presented in Figure 7A and B reflect that both these hits exerted a strong protective effect on the worms and 100\% of worms were rescued from the lethal infection throughout the experiment (over 5 days). Based on this, we propose that UE-08 and UC-15 extend the lifespan of worms during a $S$. aureus infection through their antimicrobial activity against $S$. aureus.

In contrast, upon supplementation of the other selected extracts and compounds into the screening media, we observed a cloudy appearance (similar to the untreated control) of the media indicating continued bacterial growth. We confirmed our observations by measuring the turbidity at $600 \mathrm{~nm}$. Nevertheless, addition of these extracts and compounds significantly delayed the killing of worms by $S$. aureus. Data for lifespan extension by representative hits (extracts UE-01-2 \& UE-03-5 and compounds UC-10 \& UC-12) that exhibited this phenomenon are presented in Figure 7. All these hits either did not kill $S$. aureus or only inhibited $S$. aureus growth at very high concentrations (Table 3). As they did not affect $S$. aureus viability at the tested concentrations, we propose that extracts UE-01-2 \& UE-03-5 and compounds UC-10 \& UC-12 enhance the survival of $S$. aureus-infected worms using other distinct mechanisms such as reducing bacterial virulence and/or by enhancing host immunity.

\section{Positive hits exhibited different anti-S. aureus activity}

The disc diffusion assay and MIC test were used to evaluate the positive hits obtained from the anti-infective screen for their ability to inhibit $S$. aureus growth. The disc diffusion assay detected two natural extracts (UE-08 \& UE-15) and four synthetic compounds (UC-01, UC-15, UC-27 and UC-28) out of 28 positive hits, with antibacterial effect on $S$. aureus (Figure 8). To further validate the hits, we performed the broth microdilution MIC test to obtain both MIC and MBC values.

The respective $\mathrm{MIC}$ and $\mathrm{MBC}$ values of all 28 positive hits in standard medium (TS broth) and screening medium (20\% TS broth $+80 \%$ M9 medium) are presented in Table $3 \mathrm{~A}$ and $\mathrm{B}$. The negative control (DMSO) and positive control (gentamicin) were run in parallel with a recorded MIC for gentamicin towards $S$. aureus at $1.2 \mu \mathrm{g} /$ $\mathrm{mL}$ and $\mathrm{MBC}$ of $2.4 \mu \mathrm{g} / \mathrm{mL}$. As the clearing of the liquid medium has been used as a criterion during the antiinfective screening, we also performed the MIC microdilution test using the screening medium. We found relatively similar values for both media tested. Of the positive hits screened, two natural extracts (UE-01-1 and UE-08) were found to inhibit $S$. aureus growth at a concentration of $<200 \mu \mathrm{g} / \mathrm{mL}$ while six compounds (UC-01, UC-07, UC11, UC-15, UC-27 and UC-28) exhibited bacteriostatic effects at a concentration of $\leq 200 \mu \mathrm{M}$. Of note is compound UC-15, the most potent antimicrobial agent which suppressed $S$. aureus growth at concentrations less than $62.5 \mu \mathrm{M}$ in both standard medium and screening medium. In the anti-infective screens discussed above, both $200 \mu \mathrm{g} /$ $\mathrm{mL}$ natural extracts and $200 \mu \mathrm{M}$ synthetic compounds were the working concentrations used. A further five natural extracts (UE-01-2, UE-01-6, UE-01-7, UE-15 and UE-18) and seven synthetic compounds (UC-02, UC-03, UC-06, UC-08, UC-09, UC-12 and UC-16) affected S. aureus growth in vitro but at $\sim 2$-fold higher concentrations than that used in the screen. Interestingly, seven natural extracts (UE-01-4, UE-02-4, UE-03-3, UE-03-5, UE-03-6, UE-11 and UE-12) and one synthetic compound (UC-10) which enhanced the survival of $S$. aureus-infected nematodes, did not exhibit any bacteriostatic or bactericidal activity on $S$. aureus growth in vitro. Collectively, the C. elegans model-based screen identified eight hits (29\%) that inhibited $S$. aureus growth at concentrations lower 
Table 3 In vitro MIC and MBC values of all positive extracts (A) and compounds (B) against S. aureus

\begin{tabular}{|c|c|c|c|c|c|}
\hline \multirow[t]{3}{*}{ (A) No. } & \multirow[t]{3}{*}{ Extract ID } & \multirow{2}{*}{\multicolumn{2}{|c|}{$\begin{array}{c}\text { Standard medium (TS broth) } \\
\text { Concentration }(\mu \mathrm{g} / \mathrm{mL})\end{array}$}} & \multirow{2}{*}{\multicolumn{2}{|c|}{$\begin{array}{c}\text { Screening medium (20\% TS broth }+80 \% \mathrm{M9}) \\
\text { Concentration }(\mu \mathrm{g} / \mathrm{mL})\end{array}$}} \\
\hline & & & & & \\
\hline & & MIC & MBC & MIC & MBC \\
\hline 1 & UE-01-1 & $<125$ & 1000 & $<125$ & 500 \\
\hline 2 & UE-01-2 & 2000 & - & 2000 & - \\
\hline 3 & UE-01-4 & - & - & - & - \\
\hline 4 & UE-01-6 & 2000 & - & 2000 & - \\
\hline 5 & UE-01-7 & 1000 & 2000 & 500 & 2000 \\
\hline 6 & UE-02-4 & - & - & - & - \\
\hline 7 & UE-03-3 & - & - & - & - \\
\hline 8 & UE-03-5 & - & - & - & - \\
\hline 9 & UE-03-6 & - & - & - & - \\
\hline 10 & UE-08 & $<125$ & $<125$ & $<125$ & $<125$ \\
\hline 11 & UE-11 & - & - & - & - \\
\hline 12 & UE-12 & - & - & - & - \\
\hline 13 & UE-15 & 1000 & 2000 & 500 & 2000 \\
\hline 14 & UE-18 & 1000 & - & 500 & - \\
\hline \multirow[t]{3}{*}{ (B) No. } & \multirow[t]{3}{*}{ Compound ID } & \multicolumn{2}{|c|}{ Standard medium (TS broth) } & \multicolumn{2}{|c|}{ Screening medium ( $20 \%$ TS broth $+80 \% \mathrm{M} 9$} \\
\hline & & \multicolumn{2}{|c|}{ Concentration $(\mu \mathrm{M})$} & \multicolumn{2}{|c|}{ Concentration $(\mu \mathrm{M})$} \\
\hline & & MIC & MBC & MIC & MBC \\
\hline 1 & UC-01 & 125 & 500 & 125 & 500 \\
\hline 2 & UC-02 & 2000 & 2000 & 2000 & 2000 \\
\hline 3 & UC-03 & 2000 & 2000 & 1000 & 2000 \\
\hline 4 & UC-06 & 2000 & 2000 & 1000 & 2000 \\
\hline 5 & UC-07 & 200 & 1000 & 125 & 1000 \\
\hline 6 & UC-08 & 1000 & 2000 & 1000 & 2000 \\
\hline 7 & UC-09 & 2000 & 2000 & 1000 & 2000 \\
\hline 8 & UC-10 & - & - & - & - \\
\hline 9 & UC-11 & 200 & - & 200 & - \\
\hline 10 & UC-12 & 1000 & - & 1000 & - \\
\hline 11 & UC-15 & $<62.5$ & $<62.5$ & $<62.5$ & $<62.5$ \\
\hline 12 & UC-16 & 2000 & - & 1000 & - \\
\hline 13 & UC-27 & 200 & 200 & 200 & 200 \\
\hline 14 & UC-28 & 200 & 200 & 200 & 200 \\
\hline
\end{tabular}

The negative (DMSO) and positive controls (gentamicin) were run in parallel in all replicates. The MIC of gentamicin towards S. aureus was $1.2 \mu \mathrm{g} / \mathrm{mL}$ and $\mathrm{MBC}$ was $2.4 \mu \mathrm{g} / \mathrm{mL}$.

MIC, minimum inhibitory concentration and $M B C$, minimum bactericidal concentration.

than that used in the screens, $12(42 \%)$ hits that suppressed S. aureus growth at concentrations at least twofold higher than the screen concentrations and eight hits (29\%) that had no effect on S. aureus growth in vitro even though they were effective in curing the worms from infection.

Both C. longa and curcumin demonstrated anti-S. aureus properties at different concentrations with $C$. longa inhibiting S. aureus growth at a lower concentration $(<125 \mu \mathrm{g} / \mathrm{mL})$ than curcumin $(500 \mu \mathrm{g} / \mathrm{mL})$. This suggests that the C. longa extract may contain multiple bioactive compounds that target $S$. aureus. Out of five $N$. fruticans positive samples, 4 of 5 were capable of inhibiting $S$. aureus growth with the husk methanol extract exhibiting the strongest activity.

All synthetic compounds, with the exception of UC-10, demonstrated antibacterial activity towards $S$. aureus at different concentrations. A comparison between all 14 hits and antibiotics currently used in a clinical setting did not reveal any structural relationship. However, some of the variants from these groups of compounds have previously 

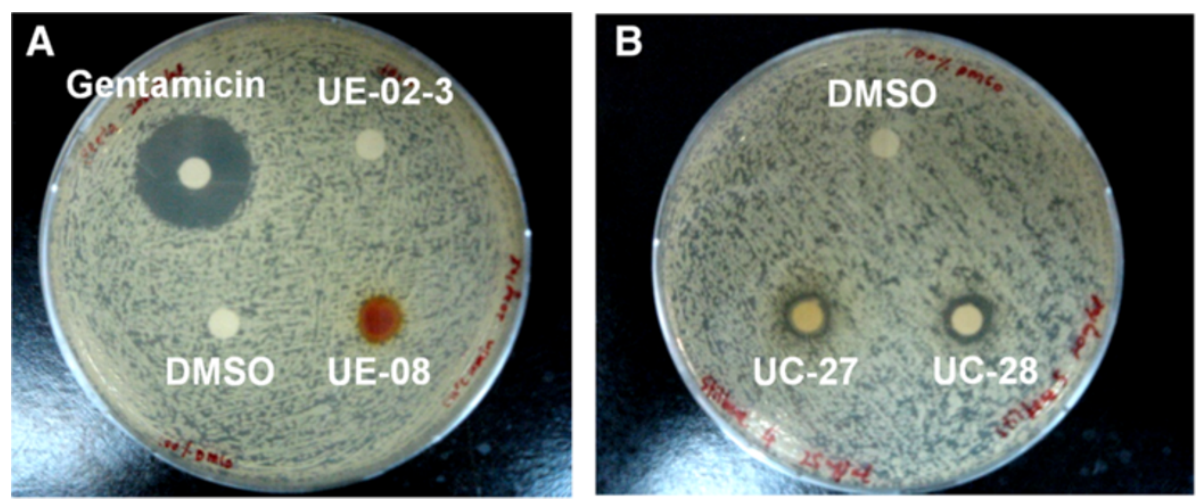

Figure 8 Inhibition of S. aureus growth by extracts and compounds. The clear zone formed around the discs indicated that $S$. aureus was susceptible to extract UE-08 (A) and compounds UC-27 \& UC-28 (B). The negative control (DMSO) showed no clear zone around the disc while the positive control $(200 \mu \mathrm{g} / \mathrm{mL}$ gentamicin) suppressed the growth of $S$. aureus and formed a $20 \mathrm{~mm}$ zone of inhibition around the disc.

been reported as potential antibacterials. UC-01, UC-02 and UC-03 are thiosemicarbazides with different elements attached to different positions of the second benzene ring and thiosemicarbazide derivatives have known antimicrobial and antifungal properties [30,31]. Derivatives of thiadiazoles and triazole-thiones (UC-06, UC-07, UC-08, UC-09, UC-11 and UC-12) also showed an inhibitory effect towards $S$. aureus at concentrations ranging from $200-2000 \mu \mathrm{M}$. Compounds containing a 1,3,4-thiadiazole nucleus and 1,2,4-trizole-thiones have a wide range of pharmacological activities including potential antimicrobial properties [32,33]. On the other hand, hydrazone derivatives are also active against $S$. aureus and $P$. aeruginosa [34]. Natural stilbenes are typical plant metabolites (e.g. resveratrol in grapes) which play a role in defending the host against microbial attack [35]. Stilbene variants such as hydroxystilbene and isopropylstilbene are potential antimicrobials and show a synergistic effect when applied together with antibiotics to inhibit and kill bacteria [36]. Our data consistently demonstrated that both stilbene derivatives (UC-27 and UC-28) showed bactericidal effect towards S. aureus at $200 \mu \mathrm{M}$. Despite ample evidence indicating the antimicrobial activity of the derivatives of these compounds, the exact mechanism on how these molecules inhibit/kill bacteria remains unknown.

\section{Positive hits also rescued worms from MRSA infection}

From the anti-infective screen and antimicrobial susceptibility test, we identified seven natural extracts (UE-01-4, UE-02-4, UE-03-3, UE-03-5, UE-03-6, UE-11 and UE-12) and one synthetic compound (UC-10) which significantly increased survival of infected nematodes without affecting S. aureus replication. The S. aureus strain NCTC8325-4 used in the screen is a methicillin-sensitive $S$. aureus (MSSA) [37]. To further explore the potency of these eight candidates, we extended our study by determining their ability in promoting the survival of MRSA-infected worms.
As the susceptibility of C. elegans towards MRSA strain ATCC33591 has not been studied before, we performed the survival assay in liquid medium to assess the virulence of this MRSA strain towards wild-type C. elegans. Our results showed that MRSA ATCC33591 killed all nematodes after 5 days post-infection with a $\mathrm{TD}_{\text {mean }}$ of $68 \pm 3.2 \mathrm{hrs}$ which is comparable to the MSSA strain NCTC8325-4 (Figure 9A). Interestingly, when we evaluated the eight hits from the MSSA screen, $5 / 8$ were also able to rescue the worms from MRSA infection. The five natural extracts that significantly promoted the survival of MRSA-infected nematodes after 72 hours infection were UE-01-4, UE-03-3, UE-03-5, UE-03-6 and UE-12 $(p<0.005)$. All five extracts contributed to more than $70 \%$ C. elegans survival upon MRSA infection compared to almost $80 \%$ death of untreated worms (Figure 9B). Specifically, the five extracts that protected the worms from both antibiotic-sensitive and antibiotic-resistant $S$. aureus infection were extracted from $N$. fruticans roots, S. macrophylla seeds and O. stamineus leaves.

The survival of MRSA-infected nematodes exposed to UE-02-4, UE-11 and UC-10 was less than 40\% (Figure 9B) indicating that these three hits were only efficacious for MSSA but not MRSA infections. Based on this, we suggest that these compounds may be able to render the antibioticsensitive strain less virulent but not the antibiotic-resistant strain. It was possible that these hits function as a specific inhibitor of virulence determinants that are complex and strain specific.

\section{Two natural extracts caused reduction in intestinal bacterial loads}

The killing of $C$. elegans by $S$. aureus is correlated with the ability of the bacteria to colonize the worm intestine [20]. S. aureus do not persist in the host, yet when the host has been in contact with the bacteria for an adequate period of time, death is observed. Similarly, 

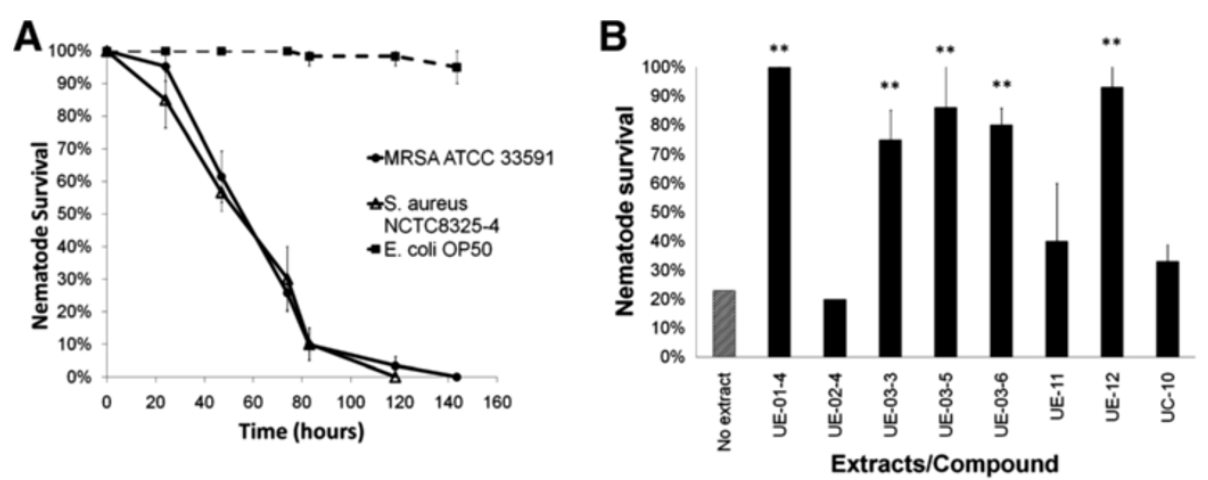

Figure 9 Nematodes infected by MRSA are rescued by natural extracts. (A) Kinetics of the killing of C. elegans by MRSA ATCC33591, S. aureus NCTC-8325-4 and E. coli OP50 in liquid medium. Graph shows the mean \pm SD of six replicates (20 worms / replicate) from a representative of three independent experiments. (B) Survival rate of MRSA-infected nematodes upon exposure to selected extracts ( $200 \mu \mathrm{g} / \mathrm{mL}) / \mathrm{compound}(200 \mu \mathrm{M})$. Graph shows the percentage of survival at 72 hours post-infection when the assay was carried out without any treatment or treatment with different extracts/ compound. Results are expressed as mean \pm SD from a representative of two individual assays. $\left({ }^{*}\right)$ denotes significant difference in percentage of survival between untreated (no extract) and treated animals $(p<0.005)$.

Pseudomonas aeruginosa PA14 also colonize and distend the worm intestinal lumen but do not persist within the host [38]. To address the question of whether the level of live $S$. aureus in the worm intestine remained the same in the presence of extracts, we measured the number of bacteria by performing a CFU assay. In vivo CFU counts were enumerated at 12 and 24 hours when $>70 \%$ of the infected non-treated population remained alive. For this assay, we tested the eight hits that showed no interference with $S$. aureus viability in the antimicrobial test.

In the absence of extract, $S$. aureus grossly colonized the worm intestine and the in vivo CFU counts for $S$. aureusinfected worms was $6.25 \times 10^{4}$ bacteria per worm after 24 hours exposure to the pathogen. This result is consistent with the data reported for worms exposed to PA14, where the bacterial CFU counts can reach up to $10^{3}-10^{5}$ bacteria per worm [29]. Upon supplementation with extract UE-01-4 and UE-12, we observed a significant reduction in the number of bacteria harvested from the C. elegans intestine, $2.5 \times 10^{2}$ and $2.3 \times 10^{2} \mathrm{CFU} /$ worm respectively $(p<0.0001)$ (Figure 10$)$. One possible explanation for this is that the ingestion of bacteria is abated as a consequence of reduced feeding following exposure to a specific bioactive molecule. We tested this hypothesis by counting the pharyngeal pumping rate of the worms in the presence of UE-01-4, as an indirect measure of the intake of bacteria. In the presence of UE-01-4, pharyngeal pumping rates were similar to untreated worms (Additional file 4). Hence, suffice to say, the low number of intestinal bacteria is not a consequence of a decrease in bacteria consumption, but rather, due to other in vivo factor/s that suppress the number of bacteria in the host intestine. For the other five extracts (UE-02-4, UE-03-3, UE-03-5, UE-03-6, UE-11) and compound UC-10, we did not observe any significant difference in the intestinal bacterial loads compared to the untreated animals.

The extracts from N. fruticans root (UE-01-4) and $O$. stamineus leaves (UE-12) limited the number of $S$. aureus in the host intestine, suggesting that the mode of action of these extracts likely involved activation of host immunity to reduce the bacterial numbers and thus clear the infection. For extracts UE-02-4, UE-03-3, UE-03-5, UE-03-6, UE-11 and compound UC-10, the manifestation of a healthy worm population that remained colonized by $S$. aureus proposed that these extracts/ compound might have rendered the bacteria less virulent and therefore harmless. The less virulent bacteria continued to colonize the host intestine but were unable to bring about death of the host. Alternatively, they may have also modulated the host response to tolerate chronic innocuous intestinal colonization. Both protective outcomes (healthy worms with harmless infection or healthy worms with cleared infection) may arise from either impairing the bacterial virulence or upregulating the host defenses.

\section{Discussion}

We have successfully established a liquid-based C. elegans $S$. aureus anti-infective screen platform which is relatively easy to perform, time-efficient and easily adaptable for large-scale screening of compounds of interest. This simple live animal screen enabled us to identify 14 natural extracts (from eight different plants and one marine sample) and 14 synthetic compounds that significantly extended the survival of $S$. aureus-infected worms. We also observed that five of the selected natural extracts conferred a survival advantage for nematodes infected by MRSA. In agreement with previously reported findings $[8,9]$, our results demonstrate that this 


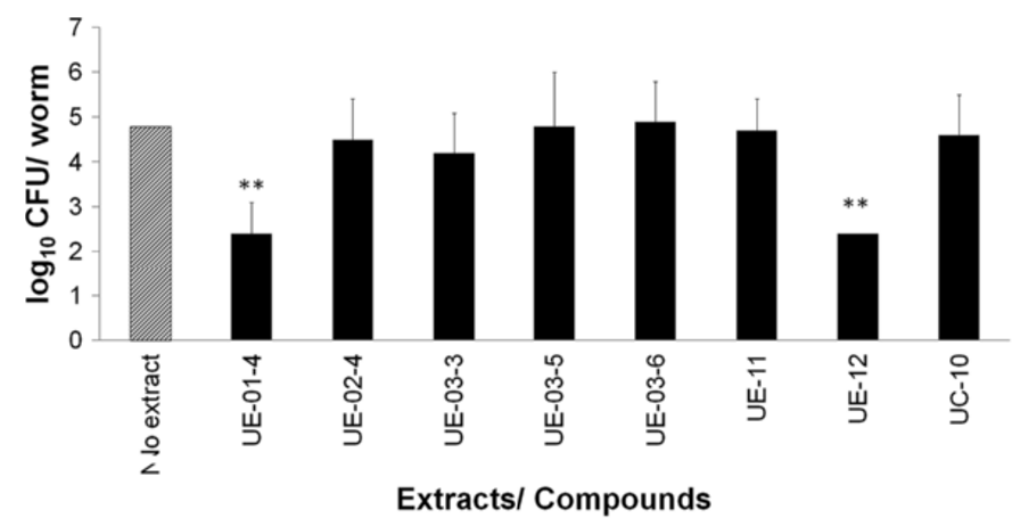

Figure 10 Extracts suppressed the number of in vivo S. aureus in infected animals. Bacterial CFU in the intestine of S. aureus-infected worms were enumerated at 24 hours post-infection in the presence and absence of extracts/compound. All extracts were tested at $200 \mu \mathrm{g} / \mathrm{mL}$ and compound was tested at $200 \mu \mathrm{M}$. The bars correspond to mean \pm SD of the number of CFU per worm from one representative of two independent replicates. $\left({ }^{* *}\right)$ marks the significant difference of in vivo CFU counts between untreated (no extract) and treated worms ( $\left.p<0.0001\right)$.

screen identifies not only substances with anti-bacterial properties, but also hits that do not affect bacterial replication in vitro which are usually overlooked in traditional in vitro cell-culture based screens.

We noticed the S. aureus- infected worms under both treated and untreated conditions appear smaller in size, similar to previously reported observations on solidmedium [22]. It is less likely that the small size of infected-worms is a result of starvation as shown by previous transcriptome analysis on fasting-response genes [22]. The smaller size of the infected worms may be a consequence of energy conservation and delayed development to compensate for the effort in fighting off the infection. Unlike the uninfected population fed on $E$. coli OP50, we observed that the surviving infected worms in the presence of most hits were also small in size and slow in development. As indicated in the antimicrobial tests, most of the positive hits did not interfere with the growth of bacteria at the concentration used in anti-infective screening $(200 \mu \mathrm{g} / \mathrm{mL})$. Due to the continuous exposure to pathogen in the screening medium, it is not surprising that the live worms resembled the phenotype of infected worms, instead of the phenotype of healthy worms fed on their natural food source.

In contrast to Salmonella typhimurium that persistently colonizes and proliferates in the nematode gut [39], S. aureus causes transient infection and continuous exposure up to a certain period of time is necessary to achieve maximal worm killing. S. aureus can grossly colonize and distend the worm intestinal lumen but does not persist within the infected host [20]. Therefore, we modified the method of Moy et al. [8] by exposing worms to live bacteria throughout the assay. A similar approach where the worms were not pre-infected has been used to screen a small molecule library for compounds that alleviate $P$. aeruginosa-mediated killing [11]. In addition, we were able to simultaneously evaluate compounds or extracts that inhibit bacterial growth and promote host survival by observing the turbidity of the liquid medium. This in turn, enables a quick selection of hits that do not affect bacterial proliferation yet enhance host survival, which will likely yield alternative therapeutics that circumvent bacterial resistance and potentially overcome the limitations of traditional antibiotics.

Among the 28 molecular entities that protected C. elegans from lethal infection by $S$. aureus, seven natural extracts and 13 synthetic compounds contain antibacterial properties against $S$. aureus at different concentrations. As expected, both C. longa and curcumin were able to inhibit $S$. aureus growth as previously described $[40,41]$. On the other hand, S. macrophylla leaves were previously reported to be active against S. aureus [42] but we show that the seed extracts did not affect $S$. aureus growth. Notably, some extracts and compounds demonstrated antibacterial effect at very high concentration (MIC: $2000 \mu \mathrm{g} / \mathrm{mL}$ ). Although they are potentially interesting as 'probe' molecules, these compounds have limited potential for commercial drug development in their current form.

Of all synthetic compounds tested, 24/29 contain a BHT moiety (Figure 1) in their chemical structure [25]. Since the compounds tested are related to this structure, it is not surprising that the hit rate was high among the synthetic compounds. However, these hits do exhibit different activities suggesting that they may have multiple targets, some of which are not in common and to which they exhibit differential affinity. Up to 13/14 of the hits demonstrated either bacteriostatic or bactericidal effects against $S$. aureus at different concentrations. BHT is a phenolic antioxidant which consists of a hydroxyl group $(-\mathrm{OH})$ bonded directly to an aromatic hydrocarbon 
group. Other substances that exert comparable free radical scavenging anti-oxidant activity as BHT have been reported to have good antibacterial and antifungal activity $[43,44]$. Phenolic compounds have also been reported to exhibit antibacterial activity towards gram positive and gram negative bacteria $[45,46]$. The mechanism thought to be responsible for phenolic toxicity to bacteria includes forming hydrogen bonds with vital proteins such as microbial enzymes [47]. Thus, the anti- S. aureus property of all the synthetic compounds tested in this study may be associated with the presence of the phenolic moiety in the BHT chemical structure. Moreover, the backbone structures of the compounds (thiosemicarbazides, thiadiazoles, triazolethiones and hydrazones) have been reported to possess antimicrobial activity against both gram positive and negative bacteria $[31,32,34]$. This is believed to be able to elevate the antimicrobial properties of these compounds.

Among all synthetic compound hits, UC-10 appeared to be particularly interesting as it rescued the infected worms without affecting $S$. aureus growth. UC-10 is a derivative of 4-(substituted benzylideneamino)-1,2,4-triazole-5-thione. In terms of structure, UC-10 differs from UC-11 and UC-12 at the second benzene ring, at which a $-\mathrm{OH}$ group was substituted at the ortho position. The different activity of this compound, as compared to the rest of the compounds, may be attributed to the presence of this additional $-\mathrm{OH}$ group and two butyl groups, resulting in two $-\mathrm{OH}$ groups flanking by tert-butyl groups into one structure (Figure 6). Modification of this aromatic ring diminished the antibacterial property of the 4-(substituted benzylideneamino)-1,2,4triazole-5-thiones compound fused to the BHT moiety. Unexpectedly, this has resulted in its ability to rescue the infected host via a distinct mechanism, probably by targeting bacterial virulence or host immunity.

Some extracts and compounds were able to promote nematode survival at concentrations much lower than their respective MIC values. The identification of seven extracts and one compound that do not interfere with bacteria replication suggests that this group of substances may apply non-conventional strategies to permit host survival whilst not affecting cell viability. These compounds may attenuate bacterial factors essential for infection such as virulence determinants involved in host damage and disease [4]. Additionally, some of these substances could modulate host responses towards the pathogen [48] or act as an immunostimulator [49] that induces the host immune defense to get rid of infection.

One of the most significant findings is the three different extracts from S. macrophylla seed (UE-03-3, 03-5, 03-6) that prolonged the survival of both $S$. aureus and MRSA-infected nematodes without diminishing the degree of $S$. aureus accumulation in the C. elegans gut. A similar effect was demonstrated in the study by Dharmalingam et al. [24] where the ethyl acetate extract (UE-03-5) also protected $C$. elegans from a lethal $P$. aeruginosa infection without affecting bacterial viability and in vivo proliferation of PA14 [24]. The fact that this extract permitted the surviving host to harbor a substantial bacterial load in the gut following infection by $S$. aureus and $P$. aeruginosa indicates that this extract might have attenuated a bacterial virulence factor or pathway which is common among pathogens. Hence, the anti-infectives identified using the C. elegans screen may be multifunctional and able to target a broad range of human pathogens. With the existence of universal virulence strategies employed by different pathogens and the conservation of innate immunity across phyla, antiinfective screening using the $C$. elegans model has the potential to identify hits that are efficacious against different groups of pathogens.

Interestingly, extracts from $N$. fruticans (UE-01-4) and O. stamineus (UE-12), plants widely found in South East Asia, both demonstrated good anti-infective effects. Although the mangrove palm is well-known for its traditional use by the local practitioners to treat different ailments in Asia, the pharmacological effect of $N$. fruticans remained poorly defined until 2011 when a group from Bangladesh reported that the leaf and stem of $N$. fruticans exhibited anti-hyperglycemic and antinociceptive activities in a mouse model [50]. On the other hand, O. stamineus leaf extract is widely used as an herbal remedy for kidney and urinary tract disorders. Previous studies revealed the anti-oxidant [51,52], anti-apoptotic [51], hepatoprotective [52] and gastroprotective [53] properties and also identified the major active fractions include eupatorin, sinensetin, 3'-hydroxy-5, 6, 7,4'-tetramethoxyflavone [54] and rosmarinic acid [55]. N. fruticans and O. stamineus extracts enabled the host to survive both antibiotic-sensitive and antibiotic-resistant $S$. aureus infection without interfering with bacterial viability, suggesting that these extracts possess compounds capable of targeting the host defense mechanism or attenuating bacterial virulence traits. The fact that the intestinal colonization of $S$. aureus was blocked after exposure to these extracts further consolidates the possibility that these compounds enhance the host immunity to eliminate the pathogen or target the bacteria factor/s that prevent them from accumulating in the intestine. Therefore, extracts UE-01-4 and UE-12 are potential anti-infective candidates that may act by targeting the host immune defenses. Further investigations are ongoing to identify the active compound/s that actively promotes lifespan extension in S. aureus-infected nematodes and the potential anti-infective underlying mechanism(s) of these candidates.

Not surprisingly, a small number of compounds accelerated the killing of nematodes by $S$. aureus. When the worms were exposed to these substances and fed with heat-killed E. coli OP50, a significant decrease in survival 
was observed as early as 24 hrs post-treatment compared to the untreated control implying that the substance is toxic to the host (Additional file 5). Hence, by using this C. elegans screen, we are able to filter compounds that exhibit toxicity upon the host [56].

\section{Conclusion}

The results of the present study demonstrate the ability of this liquid-based screen to identify anti-infective substances that enhance host survival without interfering with bacterial viability. There is an ever-growing list of Gram-positive, Gram-negative and fungal pathogens that are known to infect $C$. elegans, many of which are of clinical relevance. With minor modifications and optimization, this platform can be adapted to screen for novel therapeutic agents against infectious agents. Furthermore, by utilising readily available mutant and reporter worm strains, the mechanism of action associated with the increased survival upon treatment can be further investigated.

\section{Additional files}

Additional file 1: The source and voucher number of plant materials/marine sample for all extracts provided by the Institute for Pharmaceuticals and Neutraceuticals Malaysia (IPharm) and Universiti Malaysia Terengganu.

Additional file 2: Detailed information of all natural extracts and synthetic compounds used in the anti-infective screening.

Information of all natural extracts and synthetic compounds were presented along with the results summary of disc diffusion assay and MIC test.

Additional file 3: Screening protocol. (A) Summary of the antiinfective liquid screen towards $S$. aureus in a C. elegans model used in this study, (B) the combination agar-liquid screen protocol used by Moy et al. [8] to screen for antimicrobials towards E. faecalis in a C. elegans model and $(C)$ the high-throughput liquid-based chemical screen to screen for compounds that attenuate $P$. aeruginosa virulence and rescue $C$. elegans from infection [11]

Additional file 4: Extract UE-01-4 had no effect on the pharyngeal pumping rate of the infected worms. The pharyngeal pumping rates of S. aureus-infected nematodes upon treatment with UE-01-4 (200 $\mu \mathrm{g} /$ $\mathrm{mL}$ ) were enumerated and compared to the untreated control. The bars correspond to mean \pm SD of the pharyngeal pumps/second from one representative of two individual replicates. No significant difference in the pumping rates between the treated and untreated worms $(p>0.005)$ was observed.

Additional file 5: Representative compounds that were toxic towards nematodes. Survival curves of C. elegans fed on heat-killed $E$. coli OP50 in the presence of two selected compounds at $200 \mu \mathrm{g} / \mathrm{mL}$. Survival of OP50-fed worms reduced significantly upon exposure to UC-13 and UC-24 $(p<0.0001)$. The graph shows the mean \pm SD of three replicates (10-15 animals) from a representative of two independent assays.

\section{Abbreviations}

NCTC: National collection of type cultures; ATCC: American type culture collection; CFU: Colony forming units; MRSA: Methicillin-resistant Staphylococcus aureus; DMSO: Dimethyl sulfoxide; TS: Tryticase soy; LB: Luria Bertani; NGM: Nematode growth medium; BHT: Butylated hydroxytoluene; MIC: Minimum inhibitory concentration; MBC: Minimum bactericidal concentration; $\mathrm{TD}_{\text {mean: }}$ Mean time to death.
Competing interests

The authors declare that they have no competing interests.

\section{Authors' contributions}

CK conceived and designed the experiments, performed the experiments, analyzed the data and prepared the manuscript including revisions. WAY and NAR were involved in synthesis of compounds and manuscript preparation. MWT and SN participated in the experimental design, data analysis and manuscript preparation. All the authors have read and approved the final manuscript.

\section{Acknowledgements}

Natural extracts were provided by the Institute for Pharmaceuticals and Neutraceuticals Malaysia (IPharm) and Universiti Malaysia Terengganu whilst the synthetic compounds were generously provided by the Department of Chemistry, University of Malaya. We are grateful to Assoc. Prof. Dr. Noraziah Mohamad Zin, and the Novel Antibiotic Research (NAR) group, Universiti Kebangsaan Malaysia for kindly providing the MRSA strain ATCC33591. We thank Prof. David Rice (University of Sheffield, UK) for his constructive comments. This project was funded by the Ministry of Science, Technology and Innovation, Malaysia. CK was supported by the MyBrain15 scholarship from the Ministry of Higher Education, Malaysia.

\section{Author details}

${ }^{1}$ School of Biosciences and Biotechnology, Faculty of Science and Technology, Universiti Kebangsaan Malaysia, 43600 UKM Bangi, Selangor, Malaysia. ${ }^{2}$ Nanotechnology \& Catalysis Research Centre (NANOCAT), Block 3A, Institute of Postgraduate Studies Building, University of Malaya, Kuala Lumpur, Malaysia. ${ }^{3}$ Department of Chemistry, Faculty of Science, University of Malaya, Kuala Lumpur, Malaysia. ${ }^{4}$ Department of Genetics and Department of Microbiology and Immunology, Stanford University School of Medicine, Stanford, CA, USA. ${ }^{5}$ Current Affiliation: Department of Infectious Diseases Genentech, 1 DNA Way, 11-316 Mail Stop 33, South San Francisco, USA.

Received: 15 August 2013 Accepted: 3 January 2014

Published: 6 January 2014

\section{References}

1. Lowy FD: Staphylococcus aureus infections. N Engl J Med 1998, 339(8):520-532.

2. Hiramatsu K, Aritaka N, Hanaki H, Kawasaki S, Hosoda Y, Hori S, Fukuchi Y, Kobayashi I: Dissemination in Japanese hospitals of strains of Staphylococcus aureus heterogeneously resistant to vancomycin. Lancet 1997, 350(9092):1670-1673.

3. Hiramatsu K: Vancomycin-resistant Staphylococcus aureus: a new model of antibiotic resistance. Lancet Infect Dis 2001, 1(3):147-155.

4. Clatworthy $A E$, Pierson $E$, Hung DT: Targeting virulence: a new paradigm for antimicrobial therapy. Nat Chem Biol 2007, 3(9):541-548.

5. Escaich S: Antivirulence as a new antibacterial approach for chemotherapy. Curr Opin Chem Biol 2008, 12(4):400-408.

6. Rasko DA, Sperandio V: Anti-virulence strategies to combat bacteria-mediated disease. Nat Rev Drug Discov 2010, 9(2):117-128.

7. Hamill P, Brown K, Jenssen H, Hancock REW: Novel anti-infectives: is host defence the answer? Curr Opin Biotechnol 2008, 19(6):628-636.

8. Moy TI, Ball AR, Anklesaria Z, Casadei G, Lewis K, Ausubel FM: Identification of novel antimicrobials using a live-animal infection model. Proc Natl Acad Sci USA 2006, 103(27):10414-10419.

9. Breger J, Fuchs BB, Aperis G, Moy TI, Ausubel FM, Mylonakis E: Antifungal chemical compounds identified using a $C$. elegans pathogenicity assay. PLOS Pathog 2007, 3(2):e18.

10. Okoli I, Coleman JJ, Tampakakis E, An WF, Holson E, Wagner F, Conery AL, Larkins-Ford J, Wu G, Stern A, et al: Identification of antifungal compounds active against Candida albicans using an improved high-throughput Caenorhabditis elegans assay. PLoS One 2009, 4(9):e7025.

11. Kirienko NV, Kirienko DR, Larkins-Ford J, Wahlby C, Ruvkun G, Ausubel FM: Pseudomonas aeruginosa disrupts Caenorhabditis elegans iron homeostasis, causing a hypoxic response and death. Cell Host Microbe 2013, 13(4):406-416.

12. Giacomotto J, Segalat L, Carre-Pierrat M, Gieseler K: Caenorhabditis elegans as a chemical screening tool for the study of 
neuromuscular disorders. Manual and semi-automated methods. Methods 2012, 56(1):103-113.

13. Moy TI, Conery AL, Larkins-Ford J, Wu G, Mazitschek R, Casadei G, Lewis K, Carpenter AE, Ausubel FM: High-throughput screen for novel antimicrobials using a whole animal infection model. ACS Chem Biol 2009, 4(7):527-533.

14. Tan MW, Rahme LG, Sternberg JA, Tompkins RG, Ausubel FM: Pseudomonas aeruginosa killing of Caenorhabditis elegans used to identify P. aeruginosa virulence factors. Proc Natl Acad Sci USA 1999, 96(5):2408-2413.

15. Begun J, Sifri CD, Goldman S, Calderwood SB, Ausubel FM: Staphylococcus aureus virulence factors identified by using a high-throughput Caenorhabditis elegans-killing model. Infect Immun 2005, 73(2):872-877.

16. Garvis S, Munder A, Ball G, de Bentzmann S, Wiehlmann L, Ewbank JJ, Tummler B, Filloux A: Caenorhabditis elegans semi-automated liquid screen reveals a specialized role for the chemotaxis gene cheB2 in Pseudomonas aeruginosa virulence. PLoS Pathog 2009, 5(8):e1000540.

17. Irazoqui JE, Urbach JM, Ausubel FM: Evolution of host innate defence: insights from Caenorhabditis elegans and primitive invertebrates. Nat Rev Immunol 2010, 10(1):47-58.

18. Mellbye $B$, Schuster M: The sociomicrobiology of antivirulence drug resistance: a proof of concept. MBio 2011, 2(5):e00131.

19. Wu K, Conly J, McClure JA, Elsayed S, Louie T, Zhang K: Caenorhabditis elegans as a host model for community-associated methicillin-resistant Staphylococcus aureus. Clin Microbiol Infect 2010, 16(3):245-254.

20. Sifri CD, Begun J, Ausubel FM, Calderwood SB: Caenorhabditis elegans as a model host for Staphylococcus aureus pathogenesis. Infect Immun 2003, 71(4):2208-2217.

21. JebaMercy G, Pandian SK, Balamurugan K: Changes in Caenorhabditis elegans life span and selective innate immune genes during Staphylococcus aureus infection. Folia Microbiol (Praha) 2011, 56(5):373-380.

22. Irazoqui JE, Troemel ER, Feinbaum RL, Luhachack LG, Cezairliyan BO, Ausubel FM: Distinct pathogenesis and host responses during infection of C. elegans by P. aeruginosa and S. aureus. PLoS Pathog 2010, 6:e1000982.

23. Tabara H, Hill RJ, Mello CC, Priess JR, Kohara Y: pos-1 encodes a cytoplasmic zinc-finger protein essential for germline specification in C. elegans. Development 1999, 126(1):1-11.

24. Dharmalingam K, Tan BK, Mahmud MZ, Sedek SA, Majid MI, Kuah MK, Sulaiman SF, Ooi KL, Khan NA, Muhammad TS, et al: Swietenia macrophylla extract promotes the ability of Caenorhabditis elegans to survive Pseudomonas aeruginosa infection. J Ethnopharmacol 2011, 139(2):657-663.

25. Fries $\mathrm{E}$, Puttmann $\mathrm{W}$ : Analysis of the antioxidant butylated hydroxytoluene (BHT) in water by means of solid phase extraction combined with GC/MS. Water Res 2002, 36(9):2319-2327.

26. Yehye WA, Abdul Rahman N, Alhadi AA, Khaledi H, Ng SW, Ariffin A: Butylated hydroxytoluene analogs: synthesis and evaluation of their multipotent antioxidant activities. Molecules 2012, 17(7):7645-7665.

27. Andrews JM: BSAC standardized disc susceptibility testing method (version 6). J Antimicrob Chemother 2007, 60(1):20-41.

28. Wiegand I, Hilpert K, Hancock RE: Agar and broth dilution methods to determine the minimal inhibitory concentration (MIC) of antimicrobial substances. Nat Protoc 2008, 3(2):163-175.

29. Ooi SK, Lim TY, Lee SH, Nathan S: Burkholderia pseudomallei kills Caenorhabditis elegans through virulence mechanisms distinct from intestinal lumen colonization. Virulence 2012, 3(6):485-496.

30. Liesen AP, de Aquino TM, Carvalho CS, Lima VT, de Araujo JM, de Lima JG, de Faria AR, de Melo EJ, Alves AJ, Alves EW, et al: Synthesis and evaluation of anti-Toxoplasma gondii and antimicrobial activities of thiosemicarbazides, 4-thiazolidinones and 1,3,4-thiadiazoles. Eur J Med Chem 2010, 45(9):3685-3691.

31. Sheikhy M, Jalilian AR, Novinrooz A, Motamedi-Sedeh F: Synthesis and in vitro antibacterial evaluation of some thiosemicarbazides and thiosemicarbazones. J Biomed Sci Eng 2010, 5:39-42.

32. Pintilie $O$, Profire $L$, Sunel $V$, Popa M, Pui A: Synthesis and antimicrobial activity of some new 1,3,4-thiadiazole and 1,2,4-triazole compounds having a D, L-methionine moiety. Molecules 2007, 12:103-113.

33. Ali TE, El-Kazak AM: Synthesis and antimicrobial activity of some new 1,3-thiazoles, 1,3,4-thiadiazoles, 1,2,4-triazoles and 1,3-thiazines incorporating acridine and 1,2,3,4-tetrahydroacridine moieties. Eur J Med Chem 2010, 1:6-11.

34. Govindasami T, Pandey A, Palanivelu N, Pandey A: Synthesis, characterization and antibacterial activity of biologically important vanillin related hydrazone derivatives. Int J Org Chem 2011, 1:71-77.

35. Aslam SN, Stevenson PC, Kokubun T, Hall DR: Antibacterial and antifungal activity of cicerfuran and related 2-arylbenzofurans and stilbenes. Microbiol Res 2009, 164(2):191-195.

36. Kumar SN, Siji JV, Nambisan B, Mohandas C: Activity and synergistic interactions of stilbenes and antibiotic combinations against bacteria in vitro. World J Microbiol Biotechnol 2012, 28(11):3143-3150.

37. Hope CK, Packer S, Wilson M, Nair SP: The inability of a bacteriophage to infect Staphylococcus aureus does not prevent it from specifically delivering a photosensitizer to the bacterium enabling its lethal photosensitization. J Antimicrob Chemother 2009, 64(1):59-61.

38. Tan MW, Mahajan-Miklos S, Ausubel FM: Killing of Caenorhabditis elegans by Pseudomonas aeruginosa used to model mammalian bacterial pathogenesis. Proc Natl Acad Sci USA 1999, 96(2):715-720.

39. Aballay A, Yorgey P, Ausubel FM: Salmonella typhimurium proliferates and establishes a persistent infection in the intestine of Caenorhabditis elegans. Curr Biol 2000, 10(23):1539-1542.

40. Kim KJ, Yu HH, Cha JD, Seo SJ, Choi NY, You YO: Antibacterial activity of Curcuma longa L. against methicillin-resistant Staphylococcus aureus. Phytother Res 2005, 19(7):599-604.

41. Moghaddam KM, Iranshahi M, Yazdi MC, Shahverdi AR: The combination effect of curcumin with different antibiotics against Staphylococcus aureus. Int J Green Pharm 2009, 3(2):141-143.

42. Tan S, Osman H, Wong K, Boey P, Padzilah I: Antimicrobial and antioxidant activities of Swietenia macrophylla leaf extracts. As J Food Ag-Ind 2009, 2(2):181-188.

43. Velazquez C, Navarro M, Acosta A, Angulo A, Dominguez Z, Robles R, Robles-Zepeda R, Lugo E, Goycoolea FM, Velazquez EF, et al: Antibacterial and free-radical scavenging activities of Sonoran propolis. J Appl Microbiol 2007, 103(5):1747-1756.

44. Pramila DM, Xavier R, Marimuthu K, Kathiresan S, Khoo ML, Senthilkumar M, Sathya K, Sreeramanan S: Phytochemical analysis and antimicrobial potential of methanolic leaf extract of peppermint (Mentha piperita: Lamiaceae). J Med Plants Res 2012, 6(2):331-335.

45. Apetrei CL, Tuchilus C, Aprotosoaie AC, Oprea A, Malterud KE, Miron A Chemical, antioxidant and antimicrobial investigations of Pinus cembra L. bark and needles. Molecules 2011, 16(9):7773-7788.

46. Curcic MG, Stankovic MS, Radojevic ID, Stefanovic OD, Comic LR, Topuzovic MD, Djacic DS, Markovic SD: Biological effects, total phenolic content and flavonoid concentrations of fragrant yellow onion (Allium flavum L.). Med Chem 2012, 8(1):46-51.

47. Scalbert A: Antimicrobial properties of tannins. Phytochemistry 1991, 30(12):3875-3883.

48. Bhavsar AP, Brown ED: The worm turns for antimicrobial discovery. Nat Biotechnol 2006, 24(9):1098-1100.

49. Pukkila-Worley R, Feinbaum R, Kirienko NV, Larkins-Ford J, Conery AL, Ausubel FM: Stimulation of host immune defenses by a small molecule protects C. elegans from bacterial infection. PLoS Genet 2012, 8(6):e1002733.

50. Reza H, Haq WM, Das AK, Rahman S, Jahan R, Rahmatullah M: Anti-hyperglycemic and antinociceptive activity of methanol leaf and stem extract of Nypa fruticans Wurmb. Pak J Pharm Sci 2011, 24(4):485-488

51. Abdelwahab SI, Mohan S, Mohamed Elhassan M, Al-Mekhlafi N, Mariod AA Abdul AB, Abdulla MA, Alkharfy KM: Antiapoptotic and antioxidant properties of Orthosiphon stamineus Benth (Cat's Whiskers): intervention in the Bcl-2-mediated apoptotic pathway. Evid Based Complement Alternat Med 2011, 2011:156765.

52. Yam MF, Basir R, Asmawi MZ, Ismail Z: Antioxidant and hepatoprotective effects of Orthosiphon stamineus Benth. standardized extract. Am J Chin Med 2007, 35(1):115-126.

53. Yam MF, Ang LF, Salman IM, Ameer OZ, Lim V, Ong LM, Ahmad M, Asmawil MZ, Basir R: Orthosiphon stamineus leaf extract protects against ethanol-induced gastropathy in rats. J Med Food 2009, 12(5):1089-1097.

54. Yam MF, Mohamed EA, Ang LF, Pei L, Darwis Y, Mahmud R, Asmawi MZ, Basir R, Ahmad M: A simple isocratic HPLC method for the simultaneous determination of sinensetin, eupatorin, and 3'-hydroxy-5,6,7,4'- 
tetramethoxyflavone in Orthosiphon stamineus extracts. J Acupunct Meridian Stud 2012, 5(4):176-182.

55. Pan Y, Abd-Rashid BA, Ismail Z, Ismail R, Mak JW, Pook PC, Er HM, Ong CE: In vitro effects of active constituents and extracts of Orthosiphon stamineus on the activities of three major human CDNA-expressed cytochrome P450 enzymes. Chem Biol Interact 2011, 190(1):1-8.

56. Squiban B, Kurz CL: C. elegans: an all in one model for antimicrobial drug discovery. Curr Drug Targets 2011, 12(7):967-977.

doi:10.1186/1472-6882-14-4

Cite this article as: Kong et al.: Discovery of potential anti-infectives against Staphylococcus aureus using a Caenorhabditis elegans infection model. BMC Complementary and Alternative Medicine 2014 14:4.

\section{Submit your next manuscript to BioMed Central and take full advantage of:}

- Convenient online submission

- Thorough peer review

- No space constraints or color figure charges

- Immediate publication on acceptance

- Inclusion in PubMed, CAS, Scopus and Google Scholar

- Research which is freely available for redistribution 